\title{
INTERPOLATION AND SAMPLING HYPERSURFACES FOR THE BARGMANN-FOCK SPACE IN HIGHER DIMENSIONS
}

\author{
JOAQUIM ORTEGA-CERDÀ, ALEXANDER SCHUSTER, AND DROR VAROLIN
}

\begin{abstract}
We study those smooth complex hypersurfaces $W$ in $\mathbb{C}^{n}$ having the property that all holomorphic functions of finite weighted $L^{p}$ norm on $W$ extend to entire functions with finite weighted $L^{p}$ norm. Such hypersurfaces are called interpolation hypersurfaces. We also examine the dual problem of finding all sampling hypersurfaces, i.e., smooth hypersurfaces $W$ in $\mathbb{C}^{n}$ such that any entire function with finite weighted $L^{p}$ norm is stably determined by its restriction to $W$.

We provide sufficient geometric conditions on the hypersurface to be an interpolation and sampling hypersurface. The geometric conditions that imply the extension property and the restriction property are given in terms of some directional densities.
\end{abstract}

\section{INTRODUCTION}

Let $\omega=\sqrt{-1} \partial \bar{\partial}|z|^{2}$ denote the standard Euclidean form in $\mathbb{C}^{n}$. Fix a smooth closed complex hypersurface $W \subset \mathbb{C}^{n}$ and a plurisubharmonic function $\varphi$ such that for some contants $C, C^{\prime}>0$,

$$
C \omega \leq \sqrt{-1} \partial \bar{\partial} \varphi \leq C^{\prime} \omega
$$

in the sense of currents. For brevity, such an estimate will sometimes be denoted $\sqrt{-1} \partial \bar{\partial} \varphi \simeq \omega$.

For $p \in[1, \infty)$, let

$$
\mathfrak{B} \mathfrak{F}_{\varphi}^{p}\left(\mathbb{C}^{n}\right):=\left\{F \in \mathscr{O}\left(\mathbb{C}^{n}\right) ; \int_{\mathbb{C}^{n}}|F|^{p} e^{-p \varphi} \omega^{n}<+\infty\right\}
$$

and

$$
\mathfrak{b f}_{\varphi}^{p}(W):=\left\{f \in \mathscr{O}(W) ; \int_{W}|f|^{p} e^{-p \varphi} \omega^{n-1}<+\infty\right\}
$$

denote the generalized Bargmann-Fock spaces of weighted $L^{p}$ holomorphic functions on $\mathbb{C}^{n}$ and $W$ respectively. When $p=+\infty$ we replace the integrals by suprema. The classical Bargmann-Fock space corresponds to the case $\varphi(z)=|z|^{2}$.

Definition. Let $W$ be a uniformly flat smooth hypersurface in $\mathbb{C}^{n}$. (See section 2.)

(i) We say $W$ is an interpolation hypersurface iffor each $f \in \mathfrak{b} \mathfrak{f}_{\varphi}^{p}(W)$ there exists $F \in \mathfrak{B F}_{\varphi}^{p}\left(\mathbb{C}^{n}\right)$ such that $F \mid W=f$.

(ii) We say $W$ is a sampling hypersurface if there is a constant $M=M(p, W)$ such that for all $F \in$ $\mathfrak{B F}_{\varphi}^{p}\left(\mathbb{C}^{n}\right)$,

$$
\frac{1}{M} \int_{\mathbb{C}^{n}}|F|^{p} e^{-p \varphi} \omega^{n} \leq \int_{W}|F|^{p} e^{-p \varphi} \omega^{n-1} \leq M \int_{\mathbb{C}^{n}}|F|^{p} e^{-p \varphi} \omega^{n}
$$

when $p<+\infty$, or a similar estimate involving suprema in place of integrals when $p=\infty$.

The goal of this paper is to find geometric sufficient conditions for a uniformly flat hypersurface $W$ to be interpolating or sampling. A key concept is given in the following definition.

The first author is supported by projects BFM2002-04072-C02-02 and 2001SGR00172

The second author is partially supported by NSF grant DMS0400909. 
Definition. Let $T \in \mathscr{O}\left(\mathbb{C}^{n}\right)$ be a holomorphic function such that $W=T^{-1}(0)$ and $d T$ is nowhere zero on $W$. For any $z \in \mathbb{C}^{n}$ and any $r>0$ consider the $(1,1)$-form

$$
\Upsilon_{W}(z, r):=\sum_{i, \bar{j}=1}^{n}\left(\frac{1}{\operatorname{Vol}(B(z, r))} \int_{B(z, r)} \frac{\partial^{2} \log |T|}{\partial \zeta^{i} \partial \bar{\zeta}^{j}} \omega^{n}(\zeta)\right) \sqrt{-1} d z^{i} \wedge d \bar{z}^{j} .
$$

Remark. Clearly the definition of $\Upsilon_{W}(z, r)$ is independent of the choice of the function $T$ defining $W$. Moreover, if $\Theta_{W}$ is the current of integration associated to $W$ then $\Upsilon_{W}$ is the average of $\Theta_{W}$ in a ball of center $z$ and radius $r>0$ :

$$
\Upsilon_{W}=\Theta_{W} * \frac{\mathbf{1}_{B(0, r)}}{\operatorname{Vol}(B(0, r))},
$$

where $\mathbf{1}_{A}$ denotes the characteristic function of a set $A$ and $*$ is convolution. Thus, in particular, the trace of $\Upsilon_{W}(z, r)$ is precisely the average area of $W$ in the ball of radius $r$ and center $z$.

A useful concept in the study of interpolation and sampling for smooth hypersurfaces is the density of these hypersurfaces. Let

$$
\varphi_{r}:=\frac{\mathbf{1}_{B(0, r)} * \varphi}{\operatorname{Vol}(B(0, r))} .
$$

Definition. The density of $W$ in the ball of radius $r$ and center $z$ is

The upper density of $W$ is

$$
D(W, z, r):=\sup \left\{\frac{\Upsilon_{W}(z, r)(v, v)}{\sqrt{-1} \partial \bar{\partial} \varphi_{r}(v, v)} ; v \in T_{\mathbb{C}^{n}, z}-\{0\}\right\} .
$$

$$
D^{+}(W):=\limsup _{r \rightarrow \infty} \sup _{z \in \mathbb{C}^{n}} D(W, z, r)
$$

and the lower density of $W$ is

$$
D^{-}(W):=\liminf _{r \rightarrow \infty} \inf _{z \in \mathbb{C}^{n}} D(W, z, r) .
$$

Remark. Observe that $D(W, z, r) \leq(1-c)$ for some $c>0$ if and only if

$$
\Upsilon_{W}(z, r) \leq(1-c) \sqrt{-1} \partial \bar{\partial} \varphi_{r}(z) .
$$

On the other hand, a lower bound for $D(W, z, r)$ tells us only that the largest eigenvalue of the form $\Upsilon_{W}(z, r)-\sqrt{-1} \partial \bar{\partial} \varphi_{r}(z)$ is uniformly positive.

Our main results can be stated as follows.

Theorem 1. Let $W$ be a uniformly flat hypersurface. If $D^{+}(W)<1$ then $W$ is an interpolation hypersurface.

Theorem 2. Let $W$ be a uniformly flat hypersurface. If $D^{-}(W)>1$ then $W$ is a sampling hypersurface.

Remark. Strictly speaking, we prove Theorem 1 only for the cases $2 \leq p \leq \infty$. We omit the range $1 \leq p<2$ because of the absence of a suitable reference for $L^{p}$ estimates for solutions of $\bar{\partial}$ in this range. However, the needed estimates are known in dimension 1 (in even greater generality) and in certain special cases, and they must surely hold in general. We give a proof for the range $1 \leq p<2$ modulo the required estimates.

The hypotheses in Theorems 1 and 2 have a geometric interpretation. For simplicity, consider the classical Fock space, which correponds to $\varphi=|z|^{2}$. Then $\Upsilon_{W}(z, r)(v, v)$ is the average number of intersections of the manifold $W$ with a complex line of direction $v$ in the ball of center $z$ and radius $r$. Thus $D^{+}(W)<1$ means that in any point $z$ and in any direction $v$ the average number of intersecting points between the manifold and a complex line in the direction of $v$ is smaller than some critical value. On the other hand $D^{-}(W)>1$ means that for any point $z$ there is a direction $v$ (which may depend on the point $p$ ) such that 
in the ball of radius $r$ and center $z$ the average number of intersections between $W$ and the complex line with direction $v$ is bigger than some critical value.

Intuitively speaking, the interpretation of our theorems is that if we want $W$ to be interpolating it must be sparse in all points and all directions, but if we want it to be sampling it must be dense in all points, but only in one direction for any given point.

The interpolation and sampling problems in the generalized Fock space have been studied previously. In dimension one there is a full description given in [BO-95] that corresponds to our Theorem 1 and Theorem 2. In dimension 1 the conditions we require are also necessary. This was proved in [OS-98]. It seems plausible that this is also the case in higher dimensions, but the question of necessity remains open.

In several complex variables, there have been many partial and related results. See for instance [BT-82], [Ber-83] or [Dem-82]. In these works hypotheses are placed on the function $T \in \mathcal{O}\left(\mathbb{C}^{n}\right)$ defining $W=$ $Z(T)$ in order that $W$ be interpolating in the sense of Berenstein-Taylor,that is to say, any holomorphic function $h$ defined on $W$ and satisfying a growth condition

$$
|h(z)| \leq C \exp (C \varphi(z))
$$

can be extended to an entire function satisfying similar bounds (perhaps with a different constant). For instance a result can be found in [BT-82] stating that $W$ is interpolating in this sense if

$$
\|\partial T\| \geq C \exp (-C \varphi) \quad \text { on } W .
$$

Our results do not involve the defining function $T$, appealing instead directly to the current of intergration defining $W$. In this sense our results are more geometric in nature.

The organization of the paper is as follows. In Section 2 we define and discuss the notion of uniform flatness. In Section 3 we define a non-positive function that is singular along the variety $W$. As in [BO-95], this function is used to modify the weight of the Bargmann-Fock space in order to apply the HörmanderBombieri-Skoda technique in the proof of Theorem 1. A central point is the use of the Newton potential in the construction. In Section 4 we prove Theorem 1. We begin with the $L^{2}$ case. Our approach is to first extend the candidate function to small neighborhoods, and then to patch together these local extensions using the solution of a Cousin I problem with $L^{p}$ bounds. To pass to $L^{p}$ we use results of Berndtsson on $L^{p}$ bounds for minimal $L^{2}$ solutions of $\bar{\partial}$. In Section 5 we prove Theorem 2. Finally, in Section 6 we give a simple application of our results to improve on known sufficient conditions for sequences to be interpolating or sampling in $\mathbb{C}^{n}, n \geq 2$.

Acknowledgement. The authors would like to thank Tamas Forgacs, Jeff McNeal and Yum-Tong Siu for stimulating and useful discussions. Some of this work was done while the first author was visiting the University of Wisconsin, the second author was visiting the University of Michigan, and the third author was visiting Harvard University and the University of Michigan. The authors wish to thank these institutions for their generous hospitality.

\section{UNIFORM FLATNESS}

We shall be interested in smooth hypersurfaces $W$ satisfying the following assumption.

(F1) There is a positive constant $\varepsilon_{o}$ such that the Euclidean neighborhood

$$
N_{\varepsilon_{o}}(W):=\left\{z \in \mathbb{C}^{n} ; \operatorname{dist}(z, W)<\varepsilon_{o}\right\}
$$

is a tubular neighborhood of $W$ in $\mathbb{C}^{n}$.

Remark. In condition (F1) we mean that $N_{\varepsilon_{o}}(W)$ is diffeomorphic to a neighborhood of the zero section in the normal bundle to $W$ in $\mathbb{C}^{n}$. 
Proposition 2.1. Let $T$ be a holomorphic function in $\mathbb{C}^{n}$ such that $W=\left\{z \in \mathbb{C}^{n} ; T(z)=0\right\}$ and $d T \mid W$ is never zero. For $\varepsilon_{o}$ sufficiently small, the map

$$
(z, t) \mapsto z+t \cdot \overline{d T(z)}
$$

gives a diffeomorphism of the set $\left\{(z, t) \in W \times \mathbb{C} ;|t \cdot \overline{d T(z)}|<\varepsilon_{o}\right\}$ onto $N_{\varepsilon_{o}}(W)$, that is holomorphic in the fiber variable $t$. In fact, for any $\varepsilon \leq \varepsilon_{o}$ we have

$$
N_{\varepsilon}(W)=\{z+t \cdot \overline{d T(z)} ; z \in W,|t \cdot \overline{d T(z)}|<\varepsilon\},
$$

and thus $N_{\varepsilon}(W)$ is foliated by linear analytic disks with Euclidean circles for boundaries, and of area $\pi \varepsilon^{2}$.

Proof. Choose $p_{o} \in \partial N_{\varepsilon_{o}}(W)$. Since $W$ is a tubular neighborhood, there is a unique point $z_{o}$ such that $\operatorname{dist}\left(p_{o}, W\right)=\operatorname{dist}\left(p_{o}, z_{o}\right)$. The line connecting $p_{o}$ to $z_{o}$ is perpendicular to $W$. Indeed, if $\gamma(x)$ is a curve on $W$ with $\gamma(0)=z_{o}$, then $f(x)=\left|\gamma(x)-p_{o}\right|^{2}$ has a minimum at $x=0$. By differentiating at $x=0$ we have

$$
2 \operatorname{Re}\left\langle\gamma^{\prime}(0), z_{o}-p_{o}\right\rangle=0,
$$

and the real part of the Hermitian inner product is the Euclidean inner product. By rotational invariance of the Hermitian inner product, the whole complex line passing through $p_{o}$ and $z_{o}$ is orthogonal to $W$. Thus the points $z_{o}+e^{\sqrt{-1} \theta}\left(p_{o}-z_{o}\right)$ all minimize the distance from $\partial N_{\varepsilon_{o}}(W)$ to $z_{o}$. Now, the vector $\overline{d T(z)}$ is orthogonal to $T_{W, z_{o}}$. Indeed, if $v \in T_{W, z_{o}}$, consider any curve $\mu(x)$ such that $\mu([-\delta, \delta]) \subset W, \mu(0)=z_{o}$ and $\mu^{\prime}(0)=v$. Differentiation at $x=0$ yields

$$
0=\left.\frac{d}{d x}\right|_{x=0} T(\mu(x))=\left\langle v, \overline{d T\left(z_{o}\right)}\right\rangle
$$

It follows that any point on the circle

$$
\left\{z_{o}+\overline{t \cdot d T\left(z_{o}\right)} ;\left|t \cdot \overline{d T\left(z_{o}\right)}\right|=\varepsilon\right\}
$$

is of minimal distance $\varepsilon$ to $z_{o}$. It is now clear that the map $(z, t) \mapsto z+t \overline{d T(z)}$ is a diffeomorphism onto $N_{\varepsilon_{o}}(W)$.

Definition. A smooth hypersurface $W$ satisfying $(\mathrm{F} 1)$ is said to be uniformly flat.

If we want to extend functions to $\mathbb{C}^{n}$, uniform flatness seems a reasonable condition; we don't want points that are very far apart in $W$ to be very close to each other in the ambient space. When $n=1, W$ is a discrete set, which is uniformly flat if and only if it is uniformly separated.

For each $w \in W$, denote by $T_{W, w}$ the tangent space to $W$ at $w$ and by $n_{w}$ a unit normal to $T_{W, w}$ in the Euclidean metric. Note that $n_{w}$ is determined uniquely up to a unimodular constant. Write

$$
D_{W}(w, \varepsilon):=\left(T_{W, w} \cap B(w, \varepsilon)\right) \times\left\{\zeta n_{w} ;|\zeta|<\varepsilon\right\}
$$

for the product of the $\varepsilon$-ball centered at the origin in $T_{W, w}$, with the $\varepsilon$-disc centered at the origin of $T_{\mathbb{C}^{n}, w}$ and perpendicular to $T_{W, w}$.

Proposition 2.2. Let $W \subset \mathbb{C}^{n}$ be a uniformly flat hypersurface, and let $\varepsilon_{o}$ be such that $N_{\varepsilon_{o}}(W)$ is a tubular neighborhood. Then the following hold.

(A) Assume $n \geq 2$. For all $w \in W, W \cap D_{W}\left(w, \varepsilon_{o}\right)$ is given as a graph over $T_{W, w} \cap B\left(w, \varepsilon_{o}\right)$ by a function $y=f(x)$, where $f: T_{W, w} \cap B\left(w, \varepsilon_{o}\right) \rightarrow \mathbb{C}$ satisfies

$$
|f(w+x)| \leq \frac{|x|^{2}}{\varepsilon_{o}} .
$$

(B) For each $R>0$ there is a constant $C_{R}>0$ such that for all $z \in \mathbb{C}^{n}$,

$$
\operatorname{Area}(W \cap B(z, R)) \leq C_{R} .
$$


Proof.

(A) We may assume that $w=0$. Let us write the coordinates $z$ in $\mathbb{C}^{n}$ as $z=(x, y)$ where $x \in T_{W, 0}$ and $y \in \mathbb{C} n_{0}$. Let $A_{\varepsilon_{o}}=B_{\varepsilon_{o}} \times D_{\varepsilon_{o}}$, where $B_{\varepsilon_{o}}=\left\{|x|<\varepsilon_{o}\right\}$ and $D_{\varepsilon_{o}}=\left\{|y|<\varepsilon_{o}\right\}$. We claim first that $W \cap A_{\varepsilon_{o}}$ is a graph over $B_{\varepsilon_{o}}$. To prove this claim, write $W \cap A_{\varepsilon_{o}}=W_{o} \cup \tilde{W}$, where $W_{o}$ is the component of $W \cap A_{\varepsilon_{o}}$ containing 0 , and $\tilde{W}=\left(W \cap A_{\varepsilon_{o}}\right) \backslash W_{o}$. All of the points $\left(0, e^{\sqrt{-1} \theta} \varepsilon_{o}\right)$ are of distance at least $\varepsilon_{o}$ from $\tilde{W}$, and thus $\tilde{W}$ is confined to the "hourglass-shaped" region

$$
\left\{(x, y) \in A_{\varepsilon_{o}} ;|x|^{2}+\left|y-e^{\sqrt{-1} \theta} \varepsilon_{o}\right|^{2}>\varepsilon_{o}^{2} \text { for all } \theta \in \mathbb{R}\right\} .
$$

It follows that $\tilde{W}$ cannot intersect $B_{\varepsilon_{o}} \times \partial D_{\varepsilon_{o}}$, and must enter and exit $A_{\varepsilon_{o}}$ through $\partial B_{\varepsilon_{o}} \times D_{\varepsilon_{o}}$. Thus there must be a tangent to $\tilde{W}$ that is vertical. (Indeed, by connecting the entry and exit points with a straight line, we obtain a secant with this property, and then we can translate this vertical secant towards the origin until it becomes tangent. It must eventually become tangent because $\tilde{W}$ lies outside the hourglass, and thus does not meet the disk $\{0\} \times D_{\varepsilon_{o}}$ ). The normal to the point of vertical tangency is therefore horizontal, and must meet the disk $\{0\} \times D_{\varepsilon_{o}}$. We thus have two normals of $W$ meeting at a distance less than $\varepsilon_{o}$ to the points of $W$ from which these normals emanate. We therefore contradict the assumption that $N_{\varepsilon_{o}}(W)$ is a tubular neighborhood, unless $\tilde{W}$ is empty. Similarly, if $W_{o}$ is not a graph then it has a horizontal normal, and we arrive at the same contradiction.

Now suppose $W \cap A_{\varepsilon_{o}}$ is the graph of some function $f: B_{\varepsilon_{o}} \rightarrow D_{\varepsilon_{o}}$. (The values of $f$ are bounded by $\varepsilon_{o}$ since as we noted, $W \cap A_{\varepsilon_{o}}$ must remain outside the hourglass). Then any point $\left(0, \varepsilon_{o} e^{\sqrt{-1} \theta}\right)$ must be of distance at least $\varepsilon_{o}$ from $W$, so with $e^{\sqrt{-1} \theta}=f(x) /|f(x)|$, we have

$$
\begin{aligned}
\varepsilon_{o}^{2} & \leq|x|^{2}+\left|\varepsilon_{o} \frac{f(x)}{|f(x)|}-f(x)\right|^{2} \\
& =|x|^{2}+\left.\left|\varepsilon_{o}-\right| f(x)\right|^{2} \\
& =|x|^{2}+\varepsilon_{o}^{2}+|f(x)|^{2}-2 \varepsilon_{o}|f(x)| \\
& \leq|x|^{2}+\varepsilon_{o}^{2}-\varepsilon_{o}|f(x)|,
\end{aligned}
$$

and thus

$$
|f(x)| \leq \frac{|x|^{2}}{\varepsilon_{o}}
$$

The proof of $(\mathrm{A})$ is complete.

(B) Fix $w \in W$, which we identify with the origin in $T_{W, w}$. Let $\alpha<1$ be a positive number to be chosen shortly. From (A) we know that $W$ is a graph over $T_{W, w} \cap B\left(w, \varepsilon_{o}\right)$. Let us estimate the volume of a "thin slab" of $N_{\alpha \varepsilon_{o}}(W)$ lying over a small neighborhood $|x|<\delta$ of the origin in $T_{W, w}$, for $\delta<<\alpha \varepsilon$. To be more precise, we are estimating the volume of the region

$$
S_{\delta}\left(\varepsilon_{o}\right):=\left\{(x, f(x))+t(-\overline{d f(x)}, 1) ;|x|<\delta,|t|^{2}<\frac{\alpha^{2} \varepsilon_{o}^{2}}{|(-\overline{d f(x)}, 1)|^{2}}\right\},
$$

where $f$ is the function given by part (A). Let $\Phi(x, t)=(x, f(x))+t(-\overline{d f(x)}, 1)$. By the change of variables formula, the volume we seek is

$$
\int_{|t|<\alpha \varepsilon_{o}} \int_{|x|<\delta} \Phi^{*} d V(x, t)
$$

Now,

$$
d V(x, t)=\left(\frac{(\sqrt{-1})^{n}}{n ! 2^{n}}\right) d x_{1} \wedge d \bar{x}_{1} \wedge \ldots \wedge d x_{n-1} \wedge d \bar{x}_{n-1} \wedge d t \wedge d \bar{t} .
$$


Furthermore,

$$
\begin{aligned}
\Phi^{*} d x_{i} & =\sum_{j=1}^{n-1} \frac{\partial \Phi_{i}}{\partial x_{j}} d x_{j}+\frac{\partial \Phi_{i}}{\partial t} d t+\sum_{k=1}^{n-1} \frac{\partial \Phi_{i}}{\partial \bar{x}_{k}} d \bar{x}_{k}+\frac{\partial \Phi_{i}}{\partial t} d \bar{t} \\
& =d x_{i}-\left(\overline{\frac{\partial f}{\partial x_{i}}}\right) d t-\sum_{k=1}^{n-1} t\left(\overline{\frac{\partial^{2} f}{\partial x_{i} \partial x_{k}}}\right) d \bar{x}_{k}+0
\end{aligned}
$$

and similarly

$$
\begin{aligned}
\Phi^{*} d t & =\sum_{j=1}^{n-1} \frac{\partial f}{\partial x_{j}} d x_{j}+d t \\
\Phi^{*} d \bar{x}_{i} & =-t \sum_{j=1}^{n-1} \frac{\partial^{2} f}{\partial x_{i} \partial x_{j}} d x_{j}+d \bar{x}_{i}-\frac{\partial f}{\partial x_{i}} d \bar{t}, \text { and } \\
\Phi^{*} d \bar{t} & =\sum_{k=1}^{n-1}\left(\overline{\frac{\partial f}{\partial x_{k}}}\right) d \bar{x}_{k}+d \bar{t}
\end{aligned}
$$

It follows that $\Phi^{*} d V=J(x, t) d V$, where

$$
J(x, t)=\operatorname{det}\left(\begin{array}{c|c|c|c}
\delta_{i j} & -\left(\overline{\frac{\partial f}{\partial x_{i}}}\right) & -t\left(\overline{\frac{\partial^{2} f}{\partial x_{i} \partial x_{j}}}\right) & 0 \\
\hline \frac{\partial f}{\partial x_{j}} & 1 & 0 & 0 \\
\hline-t \frac{\partial^{2} f}{\partial x_{i} \partial x_{j}} & 0 & \delta_{i j} & -\frac{\partial f}{\partial x_{i}} \\
\hline 0 & 0 & \left(\frac{\partial f}{\partial x_{j}}\right) & 1
\end{array}\right)
$$

is the Jacobian determinant of $\Phi$. In view of the estimates on $f$ established in part (A), for $\delta$ sufficiently small the Jacobian $J$ is bounded away from zero by a constant that is independent of $w$. Indeed, as $\delta \rightarrow 0$, $J$ converges to

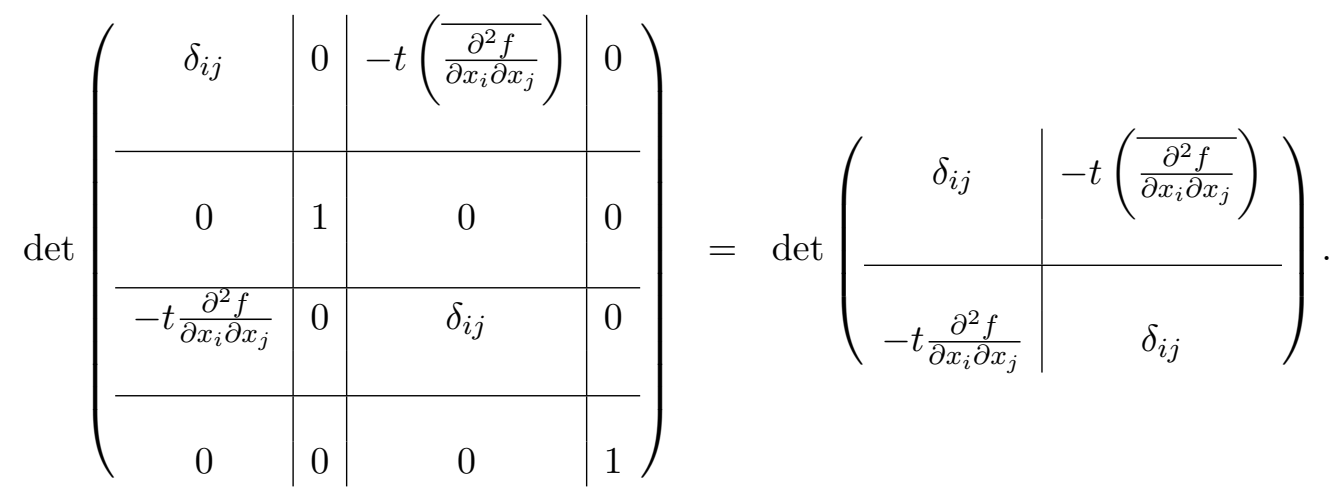

Now, from (A) we have the estimate

$$
\left|\frac{\partial^{2} f}{\partial x_{i} \partial x_{j}}\right|_{\substack{x=0 \\ 6}}^{2} \leq \frac{16}{\varepsilon_{o}},
$$


which can be obtained by simple estimates applied to

$$
\frac{\partial^{2} f}{\partial x_{i} \partial x_{j}}(0)=\lim _{h \rightarrow 0} \frac{f\left(h e_{i}+h e_{j}\right)-f\left(h e_{i}\right)-f\left(h e_{j}\right)+f(0)}{h^{2}} .
$$

Thus by choosing $\alpha>0$ sufficiently small, we have the bound

$$
J(x, t) \geq C_{o}
$$

form some $C_{o}>0$ for all $|x|<\delta$, provided $\delta$ is small enough. Note that such $\alpha>0$ need not depend on $w$. We have thus shown the following. Let $W_{\delta}=\{(x, f(x)) ;|x|<\delta\}$. Then for $\delta$ sufficiently small, there exists a constant $C>0$, depending only on the dimension $n$, such that

$$
\operatorname{Vol}\left(S_{\delta}\left(\varepsilon_{o}\right)\right) \geq C \varepsilon_{o}^{2} \operatorname{Area}\left(W_{\delta}\right) .
$$

This inequality can now be used to estimate from below the volume of much larger pieces of $N_{\varepsilon_{o} / 2}(W)$ by breaking up $W$ into very small pieces. We thus have the estimate

$$
\operatorname{Vol}\left(B\left(z, R+\alpha \varepsilon_{o}\right)\right) \geq \operatorname{Vol}\left(N_{\alpha \varepsilon_{o}}(W) \cap B(z, R)\right) \geq C \varepsilon_{o}^{2} \operatorname{Area}(W \cap B(z, R)) .
$$

The proof of $(\mathrm{B})$ is complete.

Remark. It is not hard to see that the graph of any polynomial in $(n-1)$-variables is a uniformly flat hypersurface. There are also many non-algebraic examples.

\section{Singularization OF THE WEIGHT}

As is now standard in $L^{p}$ interpolation problems in several complex variables, one needs to define a strictly plurisubharmonic weight similar to $\varphi$ with singularities along the divisor $W$. For the sampling problem, one must smooth out this weight near $W$, while maintaining global bounds away from $W$.

Our scheme for singularizing the weight follows the method of [BO-95]: we add to our weight $\varphi$ a function $s_{r}$, called the singularity, to be defined below.

To obtain good properties of the singularity, one needs to use potential theoretic aspects of the ambient space $\mathbb{C}^{n}$. For our purposes, the Newton potential plays a key role. Recall that the Newton potential is the function

$$
G(z, \zeta)=-c(n)|z-\zeta|^{2-2 n}
$$

where

$$
c(n)=\frac{1}{\pi^{n} 2^{n}(n-1)},
$$

For each $\zeta \in \mathbb{C}^{n}$, this function is harmonic in $\mathbb{C}^{n}-\{\zeta\}$ and has the property that

$$
\int_{\mathbb{C}^{n}} \sqrt{-1} \partial \bar{\partial} G(\cdot, \zeta) \wedge \omega^{n-1}=1 .
$$

The key feature making our approach possible is that this last identity involves only the trace of $\sqrt{-1} \partial \bar{\partial} G$. It is this fact precisely that links the fundamental solution of $\Delta$ to holomorphic functions on hypersurfaces.

The singularity. Consider the function

$$
\Gamma_{r}(z, \zeta):=\left(G(z, \zeta)-\frac{1}{\operatorname{Vol}(B(z, r))} \int_{B(z, r)} G(\zeta, x) \omega^{n}(x)\right) .
$$


Since $G(z, \zeta)$ is harmonic in each variable separately when $|z-\zeta|>0$, one sees immediately that $\Gamma$ is supported on the neighborhood $|z-\zeta| \leq r$ of the diagonal in $\mathbb{C}^{n} \times \mathbb{C}^{n}$. We define the singularity

$$
\begin{aligned}
s_{r}(z) & :=\int_{\mathbb{C}^{n}} \Gamma_{r}(z, \zeta) \omega^{n-1}(\zeta) \wedge(\sqrt{-1} \partial \bar{\partial} \log |T|)(\zeta) \\
& =\int_{B(z, r)} \Gamma_{r}(z, \zeta) \omega^{n-1}(\zeta) \wedge(\sqrt{-1} \partial \bar{\partial} \log |T|)(\zeta) .
\end{aligned}
$$

By the Lelong-Poincaré identity, we have

$$
s_{r}(z)=\pi \int_{W_{z, r}} G(z, \zeta) \omega^{n-1}(\zeta)-\frac{\pi}{V(r)} \int_{W_{z, r}}\left(\int_{B(z, r)} G(\zeta, x) \omega^{n}(x)\right) \omega^{n-1}(\zeta),
$$

where

$$
W_{z, r}=W \cap B(z, r) \quad \text { and } \quad V(r)=\int_{B(z, r)} \omega^{n} .
$$

Proposition 3.1. Let $T \in \mathscr{O}\left(\mathbb{C}^{n}\right)$ be a holomorphic function such that $W=\{T=0\}$ and $d T$ is nowhere zero on $W$. Then

and thus

$$
s_{r}(z)=\log |T(z)|-\frac{1}{V(r)} \int_{B(z, r)} \log |T(\zeta)| \omega^{n}(\zeta),
$$

$$
i \partial \bar{\partial} s_{r}=\Theta_{W}-\Theta_{W} * \frac{\mathbf{1}_{B(0, r)}}{\operatorname{Vol}(B(0, r))} .
$$

Proof. Let $\alpha:[0, \infty) \rightarrow[0,1]$ be a smooth compactly supported function which is identically 1 on $[0,1]$. Then for $R>>r$, we have

$$
\begin{aligned}
s_{r}(z) & =\int_{\mathbb{C}^{n}} \sqrt{-1} \partial \bar{\partial} \log |T(\zeta)| \wedge\left(\Gamma(z, \zeta) \omega^{n-1}(\zeta)\right) \\
& =\int_{\mathbb{C}^{n}} \alpha\left(R^{-2}|\zeta-z|^{2}\right) \sqrt{-1} \partial \bar{\partial} \log |T(\zeta)| \wedge\left(\Gamma(z, \zeta) \omega^{n-1}(\zeta)\right),
\end{aligned}
$$

where the second equality follows from the fact that $\Gamma(\cdot, z)$ is supported on $B(z, r)$. Integrating by parts and letting $R \rightarrow \infty$, we have

$$
\begin{aligned}
s_{r}(z)= & \int_{B(z, r)} \log |T(\zeta)| \wedge\left\{(\sqrt{-1} \partial \bar{\partial})_{\zeta} G(z, \zeta) \wedge \omega^{n-1}(\zeta)\right. \\
& \left.-\left(\frac{1}{V(r)} \int_{B(z, r)}\left[(\sqrt{-1} \partial \bar{\partial})_{\zeta} G(x, \zeta) \wedge \omega^{n-1}(\zeta)\right] \omega^{n}(x)\right)\right\} \\
= & \log |T(z)|-\left\{\int_{B(z, r)} \log |T(\zeta)|\left(\frac{1}{4 V(r)} \int_{B(z, r)} \Delta_{\zeta} G(x, \zeta) \omega^{n}(x)\right) \omega^{n}(\zeta)\right\} \\
= & \log |T(z)|-\left\{\int_{B(z, r)} \log |T(\zeta)|\left(\frac{1}{4 V(r)} \int_{B(z, r)} \Delta_{x} G(x, \zeta) \omega^{n}(x)\right) \omega^{n}(\zeta)\right\} \\
= & \log |T(z)|-\frac{1}{V(r)} \int_{B(z, r)} \log |T| \omega^{n},
\end{aligned}
$$

as desired.

We will make use of the following lemma.

Lemma 3.2. The function $s_{r}$ has the following properties. 
(a) It is non-positive.

(b) For each $r, \varepsilon>0$ there is a constant $C_{r, \varepsilon}$ such that if $\operatorname{dist}(z, W) \geq \varepsilon$, then $s_{r}(z) \geq-C_{r, \varepsilon}$.

(c) The function $e^{-2 s_{r}}$ is not locally integrable at any point of $W$.

Proof. By the sub-mean value property for subharmonic functions, $\Gamma_{r} \leq 0$, from which (a) follows. Property (c) follows immediately from Proposition 3.1.

Next, we verify that there is a constant $D_{r}$ such that for all $\zeta \in B(z, r)$,

$$
-\frac{1}{\operatorname{Vol}(B(z, r))} \int_{B(z, r)} G(\zeta, x) \omega^{n}(x) \leq D_{r} .
$$

For this, it suffices to bound the integral

$$
I_{r}(z, \zeta):=\int_{B(z, r+1)}-G(\zeta, x) \omega^{n}(x)
$$

Letting $\rho=r-|\zeta|$, we have

$$
I_{r}(z, \zeta)=\int_{B(\zeta, \rho+1)}-G(\zeta, x) \omega^{n}(x)+\int_{B(z, r)-B(\zeta, \rho+1)}-G(\zeta, x) \omega^{n}(x) .
$$

Now,

$$
\begin{aligned}
\int_{B(\zeta, \rho+1)}-G(\zeta, x) \omega^{n}(x) & =c(n) \int_{B(0, \rho+1)}|y|^{-2 n+2} \omega^{n}(y) \\
& =2 \tilde{c}(n) \int_{0}^{\rho+1} t d t \\
& =\tilde{c}(n)(\rho+1)^{2} \leq \tilde{c}(n)(r+1)^{2} .
\end{aligned}
$$

On the other hand,

$$
\int_{B(z, r)-B(\zeta, \rho+1)}-G(\zeta, x) \omega^{n}(x) \leq c(n) \int_{B(z, r+1)} \omega^{n}
$$

which demonstrates the bound for $I_{r}$.

If we now look at $z$ such that $|z-\zeta| \geq \varepsilon$ for all $\zeta \in W$, (b) follows from the above bound for $I_{r}$ together with formula (3).

\section{INTERPOLATION: THE PROOF OF THEOREM 1}

Since we assume that $i \partial \bar{\partial} \varphi \simeq \omega$, it follows that $\left|\varphi_{r}-\varphi\right| \leq C_{r}$ and therefore the spaces $\mathfrak{B F} \mathfrak{F}_{\varphi}^{p}$ and $\mathfrak{B} \mathfrak{F}_{\varphi_{r}}^{p}$ are the same space with equivalent norms. The same happens with $\mathfrak{b} \mathfrak{f}_{\varphi}^{p}$ and $\mathfrak{b} \mathfrak{f}_{\varphi_{r}}^{p}$. Therefore the manifold $W$ is interpolating (or sampling) for $\mathfrak{B} \mathfrak{F}_{\varphi}^{p}$ iff it is interpolating for $\mathfrak{B} \mathfrak{F}_{\varphi_{r}}^{p}$.

By definition of the density hypothesis, there is a sufficiently large $r$ so that

$$
\sqrt{-1} \partial \bar{\partial}\left(\varphi_{r}-\frac{\mathbf{1}_{B(0, r)}}{\operatorname{Vol}(B(0, r))} * \log |T|\right) \simeq I d .
$$

We fix this $r$ for the remainder of the proof. In principle, rahter than (4) we should be using

$$
\sqrt{-1} \partial \bar{\partial}\left(\varphi-\frac{\mathbf{1}_{B(0, r)}}{\operatorname{Vol}(B(0, r))} * \log |T|\right) \simeq I d .
$$

Thus we will actually prove that $W$ is interpolating for $\mathfrak{B} \mathfrak{F}_{\varphi_{r}}^{p}$ and by the comment above $W$ is then interpolating for $\mathfrak{B} \mathfrak{F}_{\varphi}^{p}$. 
Local extension. Let $\varphi$ be a plurisubharmonic function in $\mathbb{C}^{n}$ with $\sqrt{-1} \partial \bar{\partial} \varphi \leq M \omega$ for some $M>0$.

Proposition 4.1. Let $W$ be uniformly flat with a tubular neighbourhood of thickness bigger than $2 \varepsilon$, and choose $p \in(0, \infty]$. Then there is a constant $C=C(M, p, \varepsilon)$ such that the following holds. For any $w \in W$ and any function $f$ that is holomorphic on $W \cap B(w, 2 \varepsilon)$ there exists a function $F$ that is holomorphic on $B(w, \varepsilon)$, coincides with $f$ on $W \cap B(w, \varepsilon)$, and satisfies the estimate

$$
\int_{B(w, \varepsilon)}|F|^{p} e^{-p \varphi} \omega^{n} \leq C \int_{W \cap B(w, 2 \varepsilon)}|f|^{p} e^{-p \varphi} \omega^{n-1} .
$$

For any $0<p \leq \infty$. (If $p=\infty$, then the integrals should replaced by suprema.)

Proof. For any $w \in W$, after a rotation, we may assume that in the ball $B(w, 2 \varepsilon)$ the manifold is given by the graph of a function $y=f\left(x-w^{\prime}\right)+w_{n}$, where $w^{\prime}=\left(w_{1}, \ldots, w_{n-1}\right)$, and $f: \mathbb{C}^{n-1} \rightarrow \mathbb{C}$ with estimates $|f(x)| \leq C|x|^{2}$, by Proposition 2.2(A). For any point $z$ in the ball $B(w, 2 \varepsilon)$ we denote by $\pi(z)$ the point in $W$ with coordinates $\left(z^{\prime}, w_{n}+f\left(x-z^{\prime}\right)\right)$. The map $\pi$ is holomorphic. Let $Q_{\varepsilon}$ be the inverse image of $B(w, 2 \varepsilon) \cap W$ under $\pi$. Clearly $B(w, \varepsilon) \subset Q_{\varepsilon} \subset B(w, 3 \varepsilon)$. We shall now extend $f$ from $W \cap B(w, \varepsilon)$ to a function $F$ that is holomorphic in $Q_{\varepsilon}$ and satisfies the estimate

$$
\int_{Q_{\varepsilon}}|F|^{p} e^{-p \varphi} \omega^{n} \leq C \int_{W \cap B(w, 2 \varepsilon)}|f|^{p} e^{-p \varphi} \omega^{n-1} .
$$

The extension is constructed as follows. In $B(w, 3 \varepsilon)$ there is a bounded plurisubharmonic function $u$ such that

(i) $u$ is bounded in $B(w, 3 \varepsilon)$ by a constant depending only on $M$ and $\varepsilon$, and

(ii)

$$
\sqrt{-1} \partial \bar{\partial} u=\sqrt{-1} \partial \bar{\partial} \varphi .
$$

(For the proof, see [Lin-01, Lemma 6].) Define $h=\varphi-u$. Since $h$ is pluriharmonic there is a holomorphic function $H$ on $B(w, 3 \varepsilon)$ such that Re $H=h$. Now, for any $z \in Q_{\varepsilon}$ let $F(z):=f(\pi(z)) e^{H(z)-H(\pi(z))}$. Then $F$ is holomorphic on $Q_{\varepsilon}, F \mid\left(W \cap Q_{\varepsilon}\right)=f$, and for all $z \in Q_{\varepsilon}$ we have

$$
\begin{aligned}
|F(z)|^{p} e^{-p \varphi(z)} & =|f(\pi(z))|^{p} \exp (p h(z)-p h(\pi(z))) e^{-p \varphi(z)} \\
& =|f(\pi(z))|^{p} \exp (-p u(z)+p u(\pi(z))-p \varphi(\pi(z))) \\
& \leq C|f(\pi(z))|^{p} e^{-p \varphi(\pi(z))} .
\end{aligned}
$$

Integrating both sides over $Q_{\varepsilon}$ gives (5), and the proof is complete.

\section{Local holomorphic functions with good estimates.}

Lemma 4.2. Let $\varphi$ be a function in the unit disk $\mathbb{D}$ such that

$$
c \leq \Delta \varphi \leq \frac{1}{c} .
$$

Then there exist a constant $C>0$ and a holomorphic function $H \in \mathscr{O}(\mathbb{D})$ such that $H(0)=0$ and

$$
|\operatorname{Re} H-\varphi+\varphi(0)| \leq C .
$$

Moreover, if $\varphi$ depends on a parameter in such a way that the bound on $\Delta \varphi$ is independent of the parameter, then $H$ can be taken to depend on this parameter in such a way that $C$ does not.

The proof of this lemma, by now well known, can be found in [BO-95]. 
Construction of the interpolating function. We fix $f \in \mathfrak{b} \mathfrak{f}_{\varphi}^{p}(W)$ and $\varepsilon<\varepsilon_{0} / 2$, where $\varepsilon_{0}$ is as in Condition (F1). Take a sequence of distinct points $\left\{w_{j} ; j=1,2, \ldots\right\} \subset W$ such that

$$
N_{\varepsilon}(W) \subset \bigcup_{i=1}^{\infty}\left\{B\left(w_{i}, \frac{3}{2} \varepsilon\right)\right\}_{w_{i} \in W}
$$

and each point of $N_{\varepsilon}(W)$ is contained in at most a fixed, finite number of the sets

$$
B\left(w_{j}, 2 \varepsilon\right) \text {. }
$$

(We say that the cover is uniformly locally finite.) For convenience of notation we write $B_{i}=B\left(w_{i}, \frac{3}{2} \varepsilon\right)$. We add to the cover $\left\{B_{i}\right\}_{i \geq 1}$ another open set $B_{0}=\mathbb{C}^{n} \backslash N_{\frac{1}{2} \varepsilon}(W)$. Thus $\left\{B_{j} ; j \geq 0\right\}$ is a uniformly locally finite open cover of $\mathbb{C}^{n}$. Let $\left\{\phi_{i}\right\}_{i \geq 0}$ be a partition of unity subordinate to the cover $\left\{B_{i}\right\}$, i.e., $0 \leq \phi_{i} \leq 1, \operatorname{supp} \phi_{i} \in B_{i}$ and $\sum_{i} \phi_{i} \equiv 1$. Moreover we can assume that $\sum_{i}\left\|d \phi_{i}\right\| \leq C$.

Let $F_{i}$ denote the extension to $B_{i}$ of $f \mid W \cap B\left(w_{i}, 2 \varepsilon\right)$ given by Lemma 4.1, and set $F_{0} \equiv 0$. Since the covering $\left\{B_{i}\right\}$ is uniformly locally finite, we have

$$
\int_{\mathbb{C}^{n}} \sum_{i} \chi_{i}\left|F_{i}\right|^{p} e^{-p \varphi} \omega^{n} \lesssim \int_{W}|f|^{p} e^{-p \varphi} \omega^{n-1},
$$

where $\chi_{i}$ denotes the characteristic function of $B_{i}$ and, as usual, the symbol $\lesssim$ means that the left hand side is bounded above by a universal constant times the right hand side. We want to patch together the extensions $F_{i}$ and construct a single holomorphic extension $F$ of $f$ whose norm remains under control. In the standard language of several complex variables, we want to solve a Cousin I problem with $L^{p}$ bounds. The setup of the problem is as follows. For any pair of indices $i, j \geq 0$ we define a function $G_{i j}$ in $B_{i j}:=B_{i} \cap B_{j}$ by

$$
G_{i j}=F_{i}-F_{j} \text {. }
$$

Observe that

Finally

$$
G_{i j} \mid W \cap B_{i j} \equiv 0 \quad \text { and } \quad G_{i j}+G_{j k}+G_{k i} \equiv 0 \text { in } B_{i} \cap B_{j} \cap B_{k} .
$$

$$
\int_{\mathbb{C}^{n}} \sum_{i, j} \chi_{i} \chi_{j}\left|G_{i j}\right|^{p} e^{-p \varphi} \omega^{n} \lesssim \int_{W}|f|^{p} e^{-p \varphi} \omega^{n-1} .
$$

We seek $G_{i} \in \mathscr{O}\left(B_{i}\right)$ such that $G_{i j}=G_{i}-G_{j}$ in $B_{i j}, G_{i} \mid W \cap B_{i} \equiv 0$ and

$$
\int_{\mathbb{C}^{n}} \sum_{i} \chi_{i}\left|G_{i}\right|^{p} e^{-p \varphi} \omega^{n} \lesssim \int_{W}|f|^{p} e^{-p \varphi} \omega^{n-1} .
$$

If we find such functions $G_{i}$, then the function $F$ defined by

$$
F(x)=F_{i}(x)-G_{i}(x) \quad x \in B_{i}
$$

is an entire function. (It is well defined because $F_{i}-F_{j}=G_{i}-G_{j}$ on $B_{i j}$.) Moreover we have

$$
\left.F\right|_{W}=f \quad \text { and } \quad \int_{\mathbb{C}^{n}}|F|^{p} e^{-p \varphi} \omega^{n} \lesssim \int_{W}|f|^{p} e^{-p \varphi} \omega^{n-1} .
$$

We define $\tilde{G}_{i} \in \mathcal{C}^{\infty}\left(B_{i}\right)$ by $\tilde{G}_{i}=\sum_{j} \phi_{j} G_{i j}$. These functions have all the properties we seek, except they are not holomorphic. We shall now correct the functions $\tilde{G}_{i}$ by adding to each of them a single, globally defined function.

To this end, note that in $B_{i j}$ we have $\bar{\partial} \tilde{G}_{i}=\bar{\partial} \tilde{G}_{j}$. Thus there is a well defined $\bar{\partial}$-closed $(0,1)$-form $h$ such that

Moreover, observe that

$$
h=\bar{\partial} \tilde{G}_{i} \text { in } B_{i} .
$$

$$
\|h\| \leq \sum_{i j}\left\|\bar{\partial} \phi_{i}\right\| \cdot\left|G_{i j}\right| .
$$


Lemma 4.3. One has the estimate

$$
\int_{\mathbb{C}^{n}}\|h\|^{p} e^{-p\left(\varphi+s_{r}\right)} \omega^{n} \leq C \int_{W}|f|^{p} e^{-p \varphi} \omega^{n-1} .
$$

Proof. Recall that if $\psi$ is a weight function on the unit disk $\mathbb{D}$ such that $\Delta \psi \leq K$, then there is a constant $C$ such that for any $f \in \mathscr{O}(\mathbb{D})$,

$$
\int_{|z|<1}|f|^{p} e^{-p \psi} \leq K \int_{1 / 2<|z|<1}|f|^{p} e^{-p \psi}
$$

Indeed, the inequality is elementary in the case $\psi \equiv 0$. Since the Laplacian of $\psi$ is bounded, there is by Lemma 4.2 a non-vanishing holomorphic function $g$, such that $|g| \simeq e^{\psi}$. Thus we obtain

$$
\int_{|z|<1}|f|^{p} e^{-p \psi} \simeq \int_{|z|<1}|f / g|^{p} \leq K \int_{1 / 2<|z|<1}|f / g|^{p} \simeq \int_{1 / 2<|z|<1}|f|^{p} e^{-p \psi} .
$$

With this one variable fact it is possible to prove that

$$
\begin{aligned}
\int_{B_{i} \cap B_{j}}\left|G_{i j}\right|^{p} e^{-p\left(\varphi+s_{r}\right)} & \lesssim \int_{\left(B_{i} \cap B_{j}\right) \backslash N_{\frac{1}{2} \varepsilon}(W)}\left|G_{i j}\right|^{p} e^{-p\left(\varphi+s_{r}\right)} \\
& \simeq \int_{\left(B_{i} \cap B_{j}\right) \backslash N_{\frac{1}{2} \varepsilon}(W)}\left|G_{i j}\right|^{p} e^{-p \varphi} \\
& \lesssim \int_{\left(B_{i} \cup B_{j}\right) \cap W}|f|^{p} e^{-p \varphi} .
\end{aligned}
$$

Only the first inequality is non-trivial. To see how it follows, let $T$ be any entire function that vanishes precisely on $W$ such that $d T$ does not vanish on $W$. Then by the definition of $s_{r}$,

$$
s_{r}=\log |T|-\frac{\mathbf{1}_{B(0, r)}}{\operatorname{Vol}(B(0, r))} * \log |T| .
$$

Therefore

$$
\left|G_{i j}\right|^{p} e^{-p\left(\varphi+s_{r}\right)} \simeq\left|G_{i j} / T\right|^{p} e^{-p \psi_{r}},
$$

where

$$
\psi_{r}=\varphi-\frac{\mathbf{1}_{B(0, r)}}{\operatorname{Vol}(B(0, r))} * \log |T| .
$$

It follows by the density hypothesis that

$$
\sqrt{-1} \partial \bar{\partial} \psi_{r} \simeq I d
$$

Since the function $G_{i j} / T$ is holomorphic in $B_{i} \cap B_{j}$, we may apply the one dimensional result above. Let $U=B_{i j} \cap W$. Then $B_{i j} \simeq U \times D(0, \varepsilon)$. We integrate along the slices and apply the one-dimensional result in each disk.

By the density hypothesis, one has the inequality $\sqrt{-1} \partial \bar{\partial}\left(\varphi+s_{r}\right) \geq c \omega>0$. We will deal first with the case $p=2$. It follows from Hörmander's Theorem that there is a function $u$ such that

$$
\bar{\partial} u=h \quad \text { and } \quad \int_{\mathbb{C}^{n}}|u|^{2} e^{-2\left(\varphi+s_{r}\right)} \omega^{n} \leq C \int_{W}|f|^{2} e^{-2 \varphi} \omega^{n-1} .
$$

Moreover, the local non-integrability of $e^{-2\left(\varphi+s_{r}\right)}$ on $W$ guarantees that $u \mid W \equiv 0$. Finally, since $\varphi \geq \varphi+s_{r}$, we have that

$$
\int_{\mathbb{C}^{n}}|u|^{2} e^{-2 \varphi} \omega^{n} \leq \int_{\mathbb{C}^{n}}|u|^{2} e^{-2\left(\varphi+s_{r}\right)} \omega^{n} .
$$

It follows that the holomorphic functions $G_{i}=\tilde{G}_{i}-u$ have the desired properties. 
Next we treat the case $p \in(2, \infty]$. For ease of reading, let us write $\xi:=\varphi+s_{r}$. Assume first that $h \in L^{2}\left(e^{-\xi}\right) \cap L^{p}\left(e^{-\xi}\right)$. Let $u$ be the function of minimal norm in $L^{2}\left(e^{-\xi}\right)$ such that $\bar{\partial} u=h$. Then a Theorem of Berndtsson [Ber-97, Ber-01] states that $u$ satisfies

$$
\left\|u e^{-\varphi}\right\|_{L^{p}} \leq C_{p}\left\|h e^{-\xi}\right\|_{L^{p}}, \quad p \in[2, \infty],
$$

provided the right hand side is finite. (We point out that the constants $C_{p}$ in Berndtsson's Theorem depend only on $p$ and on the upper and lower bounds for $\sqrt{-1} \partial \bar{\partial} \varphi$.) This gives the desired bounds. Moreover, since $\left\|h e^{-\xi}\right\|_{L^{2}}<+\infty$, Hörmander's Theorem and the minimality of $u$ tell us that $\left\|u e^{-\xi}\right\|_{L^{2}}<+\infty$. Thus again $u \mid W \equiv 0$.

This proves the result for $h \in L^{2}\left(e^{-\xi}\right) \cap L^{p}\left(e^{-\xi}\right)$. To pass to the general case, instead of approximating $h$ we modify the weight $\xi$. To this end, take any sequence $\varepsilon_{j} \rightarrow 0$. Since $h$ is identically zero on a neighborhood of $W$ and $h e^{-\xi} \in L^{p}$, we have $h e^{-\varphi} \in L^{p}$. Thus once again $h e^{-\varphi} \in L^{\infty}$, and by the support of $h$ we have $h e^{-\xi} \in L^{p}$. It follows that for all $j, h e^{-\xi-\varepsilon_{j}\|z\|^{2}} \in L^{2}$. As before, the solution $u_{j}$ to $\bar{\partial} u_{j}=h$ with minimal norm in $L^{2}\left(e^{-\xi-\varepsilon_{j}\|z\|^{2}}\right)$ vanishes on $W$ and, by Berndtsson's Theorem, satisfies

$$
\left\|u_{j} e^{-\varphi-\varepsilon_{j}\|z\|^{2}}\right\|_{L^{p}} \leq C_{p}\left\|h e^{-\xi-\varepsilon_{j}\|z\|^{2}}\right\|_{L^{p}}, \quad p \in[2, \infty],
$$

where the constants $C_{p}$ are independent of $j$. It follows that $u_{j} \rightarrow u \in L^{p}\left(e^{-\varphi}\right)$. Thus we can construct holomorphic functions $F^{j}$ that extend $f$ and satisfy the estimates

$$
\int\left|F^{j}\right|^{p} e^{-p \varphi-p \varepsilon_{j}\|z\|^{2}} \leq C_{p} \int_{W}|f|^{p} e^{-p \varphi} .
$$

By a normal family argument we can take a subsequence $F^{j}$ converging to $F \in L^{p}\left(e^{-\varphi}\right)$. The convergence is unifom over compacts and thus $F$ extends $f$.

Finally we come to the case $p \in[1,2)$. Since $h$ is supported away from the singularity of $\xi$, a look at the definition of $h$ (in particular, it is constructed from certain holomorphic data and cutoff functions) shows that, since $h \in L^{p}\left(e^{-\xi}\right), h \in L^{\infty}\left(e^{-\xi}\right)$. It follows that $h \in L^{2}\left(e^{-\xi}\right)$. Let $u$ be the function of minimal norm in $L^{2}\left(e^{-\xi}\right)$ satisfying $\bar{\partial} u=h$. Unfortunately, we were unable to find a reference for the analog of Berndtsson's Theorem in the range $1 \leq p<2$. Such a theorem must certainly be true. It is true in the classical Fock space, and in dimension $n=1$ is a theorem of M. Christ [Ch-91]. (See also [MMO-03].) However, since we are unable to point to a specific reference, at the moment our proof of interpolation in the range $p \in[1,2)$ is true only modulo this result.

Let us proceed with the proof assuming that Berndtsson's Theorem holds for $p \in[1,2)$. Since $\left\|h e^{-\xi}\right\|_{L^{p}}$ is finite for $p \in[1,2]$ and $\sqrt{-1} \partial \bar{\partial} \varphi \simeq \omega, \sqrt{-1} \partial \bar{\partial} \xi \geq c \omega$ and $s_{r} \leq 0$, the hypothetical case of Berndtsson's Theorem for $p \in[1,2)$ gives the right bounds for the solution. Moreover, since $\left\|h e^{-\xi}\right\|_{L^{2}}<+\infty$, Hörmander's Theorem and the minimality of $u$ tell us once more that $\left\|u e^{-\xi}\right\|_{L^{2}}<+\infty$, and thus $u \mid W \equiv 0$.

Remark. In the $L^{2}$ case, it is possible to give an alternate proof of Theorem 1 using a modification of the method of Ohsawa-Takegoshi to the case at hand. The advantage of this approach is aesthetic; as opposed to the local approach taken in this paper, one can extend a holomorphic function from the hypersurface $W$ is one step. However, since in this paper we are interested in the $L^{p}$ case for general $p \in[1, \infty]$, we will not carry out the Ohsawa-Takegoshi type proof here. For a version on the unit ball, see [FV-05].

\section{SAMPLING}

In this section we prove Theorem 2. As in section 1 , we replace $\varphi$ by

$$
\varphi_{r}:=\frac{\mathbf{1}_{B(0, r)} * \varphi}{\operatorname{Vol}(B(0, r))}
$$

in the definition of the density and thus in the hypothesis of Theorem 2. 


\section{Restrictions and the upper sampling inequality.}

Proposition 5.1. If $W$ is a uniformly flat hypersurface, then there is a constant $C>0$ such that for all $F \in \mathfrak{B} \mathfrak{F}_{\varphi}^{p}\left(N_{\varepsilon}(W)\right)$ one has

$$
C \varepsilon^{2} \int_{W}|F|^{p} e^{-p \varphi} \omega^{n-1} \leq \int_{N_{\varepsilon}(W)}|F|^{p} e^{-p \varphi} \omega^{n} .
$$

Proof. By Proposition 2.1, $N_{\varepsilon}(W)$ is foliated by linear analytic disks with circular boundary, each of which is transverse to $W$ as well as to the boundary of $N_{\varepsilon}(W)$, has area $\pi \varepsilon^{2}$ and meets $W$ at a single point. Let $T$ be a holomorphic defining function for $W$. Making use of the diffeomorphism

$$
W \times \mathbb{D} \ni(z, t) \mapsto x+\frac{\varepsilon t \cdot \overline{d T(z)}}{|d T(z)|} \in N_{\varepsilon}(W),
$$

which is holomorphic in the second variable, we work on the product $W \times \mathbb{D}$.

Let $H(x, t)$ be the function, holomorphic in $t$, given by Lemma 4.2. That is to say,

$$
H(x, 0)=0 \quad \text { and } \quad|\operatorname{Re}(H(x, t))-\varphi(x, 0)+\varphi(x, t)| \leq C
$$

for some positive constant $C$, since we have assumed that $\sqrt{-1} \partial \bar{\partial} \varphi$ is bounded above. We then have

$$
\begin{aligned}
\varepsilon^{2}|F(x, 0)|^{p} e^{-p \varphi(x, 0)} & =\varepsilon^{2}\left|F(x, 0) e^{H(x, 0)}\right|^{p} e^{-p \varphi(x, 0)} \\
& \leq \frac{1}{2 \pi} \int_{\mathbb{D}}\left|F(x, t) e^{H(x, t)}\right|^{p} e^{-p \varphi(x, 0)} \lambda_{x}^{*} \omega \\
& \leq C \int_{\mathbb{D}}|F(x, t)|^{p} e^{-p \varphi(x, t)} \lambda_{x}^{*} \omega \\
& =C \int_{\mathcal{L}_{x}}|F|^{p} e^{-p \varphi} \omega
\end{aligned}
$$

where $\mathcal{L}_{x}=\{x+t \cdot \overline{d T(x)} ;|t \cdot \overline{d T(x)}|<\varepsilon\}$ denotes the disk through $x$. Integration over $W$ yields

$$
\varepsilon^{2} \int_{W}|F|^{p} e^{-p \varphi} \omega^{n-1} \leq C \int_{N_{\varepsilon}(W)}|F|^{p} e^{-p \varphi} \wedge \omega^{n}
$$

and the proof is complete.

Corollary 5.2. If $W$ satisfies (F1) then there is a constant $M>1$ such that for every $F \in \mathfrak{B F}_{\varphi}^{p}\left(\mathbb{C}^{n}\right)$,

$$
\int_{W}|F|^{p} e^{-p \varphi} \omega^{n-1} \leq M \int_{\mathbb{C}^{n}}|F|^{p} e^{-p \varphi} \omega^{n}
$$

\section{The proof of Theorem 2 .}

The proof will be an almost immediate application of the following sequence of definitions and lemmas.

Definition. A sequence of complex hypersurfaces $W_{n}$ is said to converge weakly to another complex hypersurface $W$ if the corresponding currents of integration $\Theta_{W_{n}}$ converge to $\Theta_{W}$ in the sense of currents.

Lemma 5.3. If $W$ is a uniformly flat complex hypersurface, then for any sequence of translations $\tau_{n}$, the sequence $W_{n}=\tau_{n}(W)$ has a subsequence converging weakly to a uniformly flat complex hypersurface $V$. Moreover, $V$ has a tubular neighborhood of at least the same thickness as that of $W$.

Proof. We denote by $\left|\Theta_{W_{n}}\right|$ the trace of the current $\Theta_{W_{n}}$. This is a positive measure that dominates all the coefficients of $\Theta_{W_{n}}$. By the uniform flatness of $W$ it is clear that for any ball $B$, $\sup _{n}\left|\Theta_{W_{n}}\right|(B)<C$ for some constant $C$ depending only on the radius of $B$. A standard compactness argument produces a subsequence that converges to a positive closed current $\theta$. It remains to show that the limit current $\theta$ is a current of integration on a manifold $V$. This is proved in [B-64], again under the assumptions that for any 
fixed ball $B$ the mass $\left|\Theta_{W_{n}}\right|(B)$ is bounded. Moreover, in this situation the support of $\Theta_{W_{n}}$ converges to $V$ and in any ball the tubular neighborhoods of the $W_{n} \cap B$ converge to a tubular neighborhood of $V \cap B$.

Definition. A sequence of plurisubharmonic functions $\varphi_{n}$ is said to converge weakly to a plurisubharmonic function $\varphi$ if the corresponding currents $\sqrt{-1} \partial \bar{\partial} \varphi_{n}$ converge to $\sqrt{-1} \partial \bar{\partial} \varphi$ in the sense of currents.

Lemma 5.4. If $\varphi$ satisfies $\sqrt{-1} \partial \bar{\partial} \varphi \simeq \omega$, then for any sequence of translations $\tau_{n}$, the sequence $\varphi_{n}=\varphi \circ \tau_{n}$ has a subsequence converging weakly to a plurisubharmonic $\psi$ and $\sqrt{-1} \partial \bar{\partial} \psi \simeq \omega$, with the constants in the estimates $\sqrt{-1} \partial \bar{\partial} \psi \simeq \omega$ controlled by the constants in the estimate $\sqrt{-1} \partial \bar{\partial} \varphi=\omega$.

Proof. This is proved in dimension 1 in [OS-98]. The same proof applies mutatis mutandi, so we content ourselves with but a sketch. Let $\theta_{n}=\sqrt{-1} \partial \bar{\partial} \varphi_{n}$. In view of the hypothesis $\sqrt{-1} \partial \bar{\partial} \varphi \lesssim \omega$, we see that $\left|\theta_{n}\right|(B(z, R)) \leq C_{R}^{n}$ where $C_{R}^{n}$ is independent of $z$, and there are functions $\psi_{n}$ such that $\sqrt{-1} \partial \bar{\partial} \psi_{n}=\theta_{n}$, $\psi_{n}(0)=0$ and $\sqrt{-1} \partial \bar{\partial} \psi_{n}$ is uniformly Lipschitz. By a normal family argument we can take a subsequence, still denoted $\psi_{n}$, such that $\psi_{n} \rightarrow \psi$ uniformly on compacts, and $\sqrt{-1} \partial \bar{\partial} \psi_{n} \rightarrow \sqrt{-1} \partial \bar{\partial} \psi$ as currents.

Definition. Given a pair $(W, \varphi)$ where $W$ is a uniformly flat complex hypersurface and $\varphi \in P S H\left(\mathbb{C}^{n}\right)$ with $\sqrt{-1} \partial \bar{\partial} \varphi \simeq \omega$, we denote by $K^{*}(W, \varphi)$ the collection of all pairs $(V, \psi)$ for which there is a sequence of translations $\tau_{n}$ such that $\tau_{n}(W)$ converge weakly to $V$ and $\varphi \circ \tau_{n}$ converge weakly to $\psi$.

Lemma 5.5. If the pair $(W, \varphi)$ satisfies $D_{\varphi}^{-}(W)=\alpha$ then all pairs $(V, \psi) \in K^{*}(W, \varphi)$ satisfy $D_{\psi}^{-}(V) \geq \alpha$

Proof. By hypothesis, for any $z \in \mathbb{C}^{n}$ and $\varepsilon>0$ there exists $r>0$ and $v \in \mathbb{C}^{n}$ of unit norm such that

$$
\int_{B(z, r)} \Theta_{W}(v, v) \geq(1-\varepsilon) \alpha \int_{B(z, r)} \sqrt{-1} \partial \bar{\partial} \varphi(v, v) .
$$

We fix an arbitrary $z \in \mathbb{C}^{n}$. Take a sequence of translations $\tau_{n}$ such that $W_{n}=\tau_{n}(W)$ and $\varphi_{n}=\tau_{n}^{*} \varphi$ converge to $V$ and $\psi$ respectively. By definition of $D_{\varphi}^{-}(W)=\alpha$, for any $\varepsilon>0$, there is an $r>0$ and unit vectors $v_{n}$ such that

$$
\int_{B(z, r)} \Theta_{W_{n}}\left(v_{n}, v_{n}\right) \geq(1-\varepsilon) \alpha \int_{B(z, r)} \sqrt{-1} \partial \bar{\partial} \varphi_{n}\left(v_{n}, v_{n}\right) .
$$

By compactness there is a subsequence of the $v_{n}$ converging to $v$ with $\|v\|=1$. By Hurwitz's theorem

and since $\sqrt{-1} \partial \bar{\partial} \varphi \simeq \omega$

$$
\liminf _{n} \int_{B(z, r)} \Theta_{W_{n}}\left(v_{n}, v_{n}\right) \leq \int_{B(z, r)} \Theta_{V}(v, v)
$$

$$
\lim _{n} \int_{B(z, r)} \sqrt{-1} \partial \bar{\partial} \varphi_{n}\left(v_{n}, v_{n}\right)=\int_{B(z, r)} \sqrt{-1} \partial \bar{\partial} \psi(v, v)
$$

Definition. The pair $(V, \psi)$ is said to be determining if for any $f \in \mathfrak{B} \mathfrak{F}_{\psi}^{\infty}\left(\mathbb{C}^{n}\right),\left.f\right|_{V}=0$ implies that $f \equiv 0$.

Lemma 5.6. The manifold $W$ is sampling for $\mathfrak{B F}_{\varphi}^{\infty}$ if all pairs $(V, \psi) \in K^{*}(W, \varphi)$ are determining.

Lemma 5.6 was essentially proved by Beurling in [Be-89, pp. 341-365], so we omit the proof. This is a key result because it allows us to determine that $W$ is sampling simply by checking the more easily verified condition that $V$ is determining.

Lemma 5.7. If $D_{\psi}^{-}(V)>1$ then the pair $(V, \psi)$ is determining.

Proof. Without loss of generality we assume that $0 \notin V$. In order to arrive at a contradiction, assume there exists an $F \in \mathfrak{B F}_{\varphi}^{\infty}$ with $F \mid V \equiv 0$ and $F(0)=1$. By hypothesis there is a direction $v$ such that the density of $V$ in the direction of $v$ is greater than 1 . We will work on the line $\ell=\mathbb{C} v$. Write $f=F \mid \ell$ and $\phi=\varphi \mid \ell$, 
and let $\Gamma=V \cap \ell$. Then $\Gamma$ is a uniformly separated sequence with density $>1$ with respect to the weight $\phi$. Recall that the one-dimensional lower density is

$$
\liminf _{R \rightarrow \infty} \inf _{z \in \ell} \frac{\#(\Gamma \cap D(z, R))}{\int_{D(z, R)} \Delta \phi} .
$$

By hypothesis $f(0)=1$. Now, if $n(0, s)$ denotes the number of zeros of $f$ in $D(0, s)$, then

$$
\liminf _{R \rightarrow \infty} \frac{n(0, R)}{\int_{D(0, R)} \Delta \phi}>1 .
$$

Applying Jensen's Formula to $f$, we get

$$
\int_{1}^{R} \frac{n(0, s)}{s} d s \leq \frac{1}{2 \pi} \int_{0}^{2 \pi} \log \left|f\left(R e^{\sqrt{-1} \theta}\right)\right| d \theta .
$$

Since $\log \left|f\left(R e^{\sqrt{-1} \theta}\right)\right| \leq \phi\left(R e^{\sqrt{-1} \theta}\right)+K$, we obtain

$$
\int_{1}^{R} \frac{n(0, s)}{s} d s \leq \frac{1}{2 \pi} \int_{0}^{2 \pi} \phi\left(R e^{\sqrt{-1} \theta}\right) d \theta+K .
$$

Now, by Green's Theorem we have

$$
\begin{aligned}
\int_{0}^{2 \pi} \phi\left(R e^{\sqrt{-1} \theta}\right) d \theta & =\int_{0}^{R} \frac{1}{s} \int_{0}^{2 \pi}\left(s \frac{\partial}{\partial s} \phi\left(s e^{\sqrt{-1} \theta}\right) d \theta\right) d s \\
& =4 \int_{0}^{R} \frac{\int_{D(0, s)} \Delta \phi}{s} d s
\end{aligned}
$$

and thus

$$
\int_{1}^{R} \frac{n(0, s)}{s} d s \leq \frac{2}{\pi} \int_{0}^{R} \frac{\int_{D(0, s)}(\Delta \phi)}{s} d s+K .
$$

Thus since $\int_{D(0, R)} \Delta \phi \simeq R^{2}$,

which contradicts (6).

$$
\frac{\int_{1}^{R} \frac{n(0, s)}{s} d s}{\int_{0}^{R} \frac{\int_{D(0, s)}(\Delta \phi)}{s} d s} \leq 1+K / R^{2}
$$

Lemma 5.8. If $W$ is a uniformly flat sampling hypersurface for $\mathfrak{B F}_{\varphi}^{\infty}$ then there is a uniformly separated sequence $\Sigma \subset W$ that is sampling for $\mathfrak{B F}_{\varphi}^{\infty}$.

Remark. The definition of a sampling sequence is given in Section 6 below.

Proof. Any set $F$ that is sampling for $\mathfrak{B F}_{\varphi}^{\infty}$ contains a uniformly separated sampling sequence. This is proved in [Lin-01, Proposition 19]. (For the 1 dimensional case, see [OS-98, Proposition 2].)

Lemma 5.9. Let $1 \leq p \leq \infty$. If $\Sigma$ is a uniformly separated sampling sequence for $\mathfrak{B F}_{\varphi+\varepsilon|z|^{2}}^{\infty}$ then it is a sampling sequence for $\mathfrak{B} \mathfrak{F}_{\varphi}^{p}$.

Proof. Denote by $\mathfrak{B F}_{\varphi+\varepsilon|z|^{2}}^{\infty, 0}$ the closed subspace of $\mathfrak{B F}_{\varphi+\varepsilon|z|^{2}}^{\infty}$ consisting of functions $f$ such that

$$
\lim _{z \rightarrow \infty}|f| e^{-\varphi-\varepsilon|z|^{2}}=0 .
$$

The restriction operator

$$
R: \mathfrak{B F}_{\varphi+\varepsilon|z|^{2}}^{\infty, 0} \rightarrow \ell_{\varphi+\varepsilon|z|^{2}}^{\infty, 0}
$$


sending $f$ to $\{f(\sigma)\}_{\sigma \in \Sigma}$ is a bounded linear operator. Since $\Sigma$ is sampling, $R$ is onto and has closed range. Thus $\mathrm{R}$ defines an isomorphism between $\mathfrak{B F}_{\varphi+\varepsilon|z|^{2}}^{\infty, 0}$ and its image. For any $z \in \mathbb{C}^{n}$ the weighted point evaluation

$$
f \mapsto f(z) e^{-\varphi(z)-\varepsilon|z|^{2}}
$$

is bounded on $\mathfrak{B F}_{\varphi+\varepsilon|z|^{2}}^{\infty, 0}$. Thus, for every $z$ there is a sequence $k(z, \sigma)$ such that

$$
f(z) e^{-\varphi(z)-\varepsilon|z|^{2}}=\sum_{\sigma \in \Sigma} k(z, \sigma) f(\sigma) e^{-\varphi(\sigma)-\varepsilon|\sigma|^{2}},
$$

for all functions $f \in \mathfrak{B F}_{\varphi+\varepsilon|z|^{2}}^{\infty, 0}$ and such that $\sum|k(z, \sigma)| \leq K$ uniformly in $z$. We fix $p \in[1, \infty)$. For an arbitrary $g \in \mathfrak{B F}_{\varphi}^{p}$ and $z \in \mathbb{C}^{n}$,

$$
f(w)=g(w) e^{2 \varepsilon w \cdot \bar{z}-\varepsilon|z|^{2}}
$$

belongs to $\mathfrak{B F}_{\varphi+\varepsilon|z|^{2}}^{\infty, 0}$ and thus we may apply (7) to obtain

$$
g(z) e^{-\varphi(z)}=f(z) e^{-\varphi(z)-\varepsilon|z|^{2}}=\sum_{\sigma \in \Sigma} k(z, \sigma) f(\sigma) e^{-\varphi(\sigma)-\varepsilon|\sigma|^{2}} .
$$

Thus

$$
|g(z)| e^{-\varphi(z)} \leq \sum_{\sigma \in \Sigma}|k(z, \sigma)||g(\sigma)| e^{-\varphi(\sigma)} e^{-\varepsilon|z-\sigma|^{2}} .
$$

This together with the inequality $\sum|k(z, \sigma)| \leq K$ implies that

$$
\int_{\mathbb{C}^{n}}|g(z)|^{p} e^{-p \varphi(z)} \lesssim \sum_{\sigma \in \Sigma}|g(\sigma)|^{p} e^{-p \varphi(\sigma)}
$$

and that

$$
\sup |g(z)| e^{-\varphi(z)} \lesssim \sup _{\sigma \in \Sigma}|g(\sigma)| e^{-\varphi(\sigma)}
$$

Lemma 5.10. Let $W$ be a uniformly flat hypersurface. Let $\Sigma$ be a uniformly separated sequence contained in $W$. If $\Sigma$ is a sampling sequence for $\mathfrak{B F}_{\varphi}^{p}$ then $W$ is a sampling hypersurface for $\mathfrak{B} \mathfrak{F}_{\varphi}^{p}$.

Proof. We only need to prove that for any $z \in W$, the inequality

$$
|f(z)|^{p} e^{-p \varphi(z)} \leq C \int_{D_{z}}|f(x)|^{p} e^{-p \varphi(x)} \omega^{n-1}(x),
$$

holds, where $D_{z}=W \cap B(z, \varepsilon)$, and the constant $C$ may depend on the radius $\varepsilon$ of the ball but not on the center $z$. For if (8) holds then for any function $f \in \mathfrak{B} \mathfrak{F}_{\varphi}^{p}$,

$$
\left\|f e^{-\varphi}\right\|_{p}^{p} \lesssim \sum|f(\sigma)|^{p} e^{-p \varphi(\sigma)} \lesssim \sum_{\sigma \in \Sigma} \int_{D_{\sigma}}|f|^{p} e^{-p \varphi} \omega^{n-1} \leq \int_{W}|f|^{p} e^{-p \varphi} \omega^{n-1} .
$$

In order to prove (8) we need the hypothesis that $i \partial \bar{\partial} \varphi \simeq \omega$. Under this hypothesis we may again invoke the existence of a non vanishing function $h \in \mathscr{O}(B(z, \varepsilon))$ such that $e^{\varphi} \simeq|h|$ in $B(z, \varepsilon)$ with constants independent of $z$. Thus, we may replace $e^{-\varphi}$ by $h^{-1}$ in (8) and get the result if we prove that

$$
|g(z)|^{p} \leq C \int_{D_{z}}|g(x)|^{p} \omega^{n-1}(x) .
$$

If $D_{z}$ is a hyperplane then the latter estimate holds for all holomorphic functions $g$ by the sub-mean value property. In a general uniformly flat hypersurface the estimate holds because the distortion introduced in $\omega^{n-1}$ upon rectifying $D_{z}$ by a change of variables is uniformly bounded due to property (A) in Lemma 2.2 for uniformly flat hypersurfaces. 
Finally, we are ready to prove Theorem 2 . To this end, let $\varepsilon>0$ be such that $D_{\varphi}^{-}(W)>1+\varepsilon$. We start by proving that $W$ is a sampling manifold for $\mathfrak{B F}_{\varphi_{\varepsilon}}^{\infty}$, where $\varphi_{\varepsilon}=\varphi+\varepsilon|z|^{2}$. In order to do so, we use Lemma 5.6. We need to check that for any pair $(V, \psi) \in K^{*}\left(W, \varphi_{\varepsilon}\right)$ the pair $(V, \psi)$ is determining. This is true in view of Lemmas 5.5 and 5.7. Now we take the sequence $\Sigma \subset W$ given by Lemma 5.8. This sequence $\Sigma$ is a sampling sequence for $\mathfrak{B F}_{\varphi_{\varepsilon}}^{\infty}$ and thus it is also sampling for $\mathfrak{B F}_{\varphi}^{p}$ by Lemma 5.9. Finally by Lemma 5.10 we conclude that $W$ is a sampling manifold for $\mathfrak{B} \mathfrak{F}_{\varphi}^{p}$.

\section{AN APPLICATION TO SEQUENCES IN HIGHER DIMENSIONS}

Let $\varphi$ be a plurisubharmonic function in $\mathbb{C}^{n}$ such that for some $c>0$

$$
c \omega \leq \sqrt{-1} \partial \bar{\partial} \varphi \leq \frac{1}{c} \omega .
$$

Let $\Gamma$ be a uniformly separated sequence of points in $\mathbb{C}^{n}$. We consider the space

$$
\ell_{\varphi}^{p}(\Gamma):=\left\{\left\{a_{\gamma}\right\}_{\gamma \in \Gamma} \subset \mathbb{C} ; \sum_{\Gamma}\left|a_{\gamma}\right|^{p} e^{-p \varphi(\gamma)}<+\infty\right\} .
$$

Recall that $\Gamma$ is an interpolation sequence if for each $\left\{a_{\gamma}\right\} \in \ell_{\varphi}^{p}(\Gamma)$ there exists $F \in \mathfrak{B} \mathfrak{F}_{\varphi}^{p}\left(\mathbb{C}^{n}\right)$ such that

$$
F(\gamma)=a_{\gamma}, \quad \gamma \in \Gamma
$$

and that $\Gamma$ is a sampling sequence if there is a constant $M>1$ such that for all $F \in \mathfrak{B F} \mathfrak{F}_{\varphi}^{p}\left(\mathbb{C}^{n}\right)$

$$
\frac{1}{M} \int_{\mathbb{C}^{n}}|F|^{p} e^{-p \varphi} \omega^{n} \leq \sum_{\Gamma}|F(\gamma)|^{p} e^{-p \varphi(\gamma)} \leq M \int_{\mathbb{C}^{n}}|F|^{p} e^{-p \varphi} \omega^{n} .
$$

Sufficient conditions are known for a sequence to be interpolating, and also sampling. There are also (different) necessary conditions. However, all the known conditions involve only the number of points of the sequence contained in a large ball. It has been known for some time that such a condition could not possibly characterize interpolation and sampling sequences, since it does not take into account how points are distributed relative to one another. For example, consider the situation of interpolation. If all the points of a sequence lie on a line, then to be interpolating there must be at most $O\left(r^{2}\right)$ points in any ball of radius $r$. On the other hand, the number of points of a lattice in $\mathbb{C}^{n}$ lying inside a ball of radius $r$ is $O\left(r^{2 n}\right)$. Thus any condition for interpolation that takes into account only the number of points of the sequence lying in a ball of radius $r$ would not suffice to conclude that any lattice, no matter how sparse, is an interpolation sequence. Similar reasoning shows that analogous problems arise in the case of sampling conditions.

The present paper and the paper [SV-03] suggest an approach to studying interpolation and sampling sequences by induction on dimension. In [SV-03] two of us tackled the 1-dimensional case. The present paper tackles the problem from the other end. In this section, we show that the results of the present paper already improve what is known for sequences in higher dimension.

6.1. Applications to interpolation. For simplicity, we restrict to the case of sequences in $\mathbb{C}^{2}$. As mentioned, at present rather poor density conditions are known in the general higher dimensional case. However, in a very symmetric situation there is a characterization of interpolation and sampling sequences in $\mathbb{C}^{2}$. Suppose the sequence $\Gamma$ is of the form

$$
\Gamma=\Gamma_{1} \times \Gamma_{2},
$$

where $\Gamma_{1}, \Gamma_{2}$ are sequences in $\mathbb{C}$. Suppose, moreover, that the weight $\varphi$ splits:

$$
\varphi(z, w)=\varphi_{1}(z)+\varphi_{2}(w),
$$

where $\Delta \varphi_{j} \simeq 1, j=1,2$. Then the following is true:

Claim. $\Gamma$ is interpolating (resp. sampling) with respect to the weight $\varphi$ if and only if for both $j=1$ and 2 , $\Gamma_{j}$ is interpolating (resp. sampling) for the weight $\varphi_{j}$. 
This result can be recovered from the 1-dimensional characterization of interpolation and sampling established in [BO-95] and [OS-98].

We shall now generalize this result to the case of arbitrary sequences lying on a family of parallel lines in $\mathbb{C}^{2}$. To this end, let $\Gamma=\left\{\gamma_{j}\right\}, \Lambda_{1}=\left\{\lambda_{1, j}\right\}, \Lambda_{2}=\left\{\lambda_{2 j}\right\}, \ldots$ be sequences in $\mathbb{C}$. Define

$$
\Sigma=\left\{\left(\gamma_{j}, \lambda_{j k}\right) ; j, k=1,2, \ldots\right\} .
$$

As a corollary of our main results, we have the following theorem.

Theorem 3. Suppose that for some fixed $\varepsilon>0$, each $\Lambda_{j}$ has density $\leq 1-\varepsilon$, with respect to the weight $\varphi\left(\gamma_{j}, \cdot\right)$, and that

$$
\frac{\# \Gamma \cap \mathbb{D}(z, r)}{r^{2} \Delta_{z} \varphi(z, w)}<\frac{\operatorname{det}(\sqrt{-1} \partial \bar{\partial} \varphi(z, w))}{\Delta_{z} \varphi(z, w) \Delta_{w} \varphi(z, w)}
$$

for all $z, w \in \mathbb{C}$. Then $\Sigma$ is interpolating for $\mathfrak{B} \mathfrak{F}_{\varphi}^{p}\left(\mathbb{C}^{2}\right)$.

Proof. Let $W=\Gamma \times \mathbb{C}$. We first calculate the density of $W$. To this end, let $T(z, w)=\sigma(z)$, where $\sigma$ is a holomorphic function whose zero set, counting multiplicity, is $\Gamma$. Then the zero set of $T$ in $\mathbb{C}^{2}$ is $W$, and one sees easily that

$$
\begin{aligned}
D(W, x, r) & =\sup _{t \in \mathbb{C}} \frac{\sum_{j} \operatorname{Area}\left(\left(\left\{\gamma_{j}\right\} \times \mathbb{C}\right) \cap B(x, r)\right)}{\operatorname{Vol}(B(x, r))\left(\Delta_{z} \varphi(x)+\Delta_{w} \varphi(x)|t|^{2}+2 \operatorname{Re}\left(\varphi_{z \bar{w}} \bar{t}\right)\right)} \\
& =\frac{\sum_{j} \operatorname{Area}\left(\left(\left\{\gamma_{j}\right\} \times \mathbb{C}\right) \cap B(x, r)\right)}{\operatorname{Vol}(B(x, r))\left(\Delta_{z} \varphi(x)-\frac{\left|\varphi_{z \bar{w}}(x)\right|^{2}}{\Delta_{w} \varphi(x)}\right)} \\
& =\frac{\sum_{j} \operatorname{Area}\left(\left\{\gamma_{j}\right\} \times \mathbb{C} \cap B(x, r)\right)}{\operatorname{Vol}(B(x, r)) \Delta_{z} \varphi(x)} \frac{\Delta_{z} \varphi(x) \Delta_{w} \varphi(x)}{\operatorname{det}(\sqrt{-1} \partial \bar{\partial} \varphi(x))} .
\end{aligned}
$$

Since we are going to take lim sup as $r \rightarrow \infty$, condition (9) implies that $W$ is an interpolation hypersurface.

Now suppose given a sequence of values $\left\{a_{j k}\right\}$ such that

$$
\sum_{j} \sum_{k}\left|a_{j k}\right|^{p} e^{p \varphi\left(\gamma_{j}, \lambda_{j k}\right)}<+\infty .
$$

Fix $j$. Since $\Lambda_{j}$ is interpolating, there is a function $g_{j}(w)$ such that

$$
g_{j}\left(\lambda_{j k}\right)=a_{j k} \quad \text { and } \quad \int_{\mathbb{C}}\left|g_{j}(w)\right|^{p} e^{-p \varphi\left(\gamma_{j}, w\right)} d A(w) \leq C \sum_{k}\left|a_{j k}\right|^{p} e^{p \varphi\left(\gamma_{j}, \lambda_{j k}\right)}
$$

for some absolute constant $C$. (This is not immediate; one has to use the fact that an interpolation operator can be constructed with norm depending only on the density of the sequence. The uniformity of $C$ now follows because the density of $\Lambda_{j}$ is bounded away from 1 uniformly in $j$.)

Define the function $f \in \mathscr{O}(W)$ by

$$
f\left(\gamma_{j}, w\right)=g_{j}(w) .
$$

Then the estimates on the $L^{p}$ norms of $g_{j}$ imply that $f \in \mathfrak{b} \mathfrak{f}_{\varphi}^{p}(W)$. By Theorem 1 , there exists $F \in \mathfrak{B} \mathfrak{F}_{\varphi}^{p}\left(\mathbb{C}^{2}\right)$ such that $F \mid W=f$. Thus

$$
F\left(\gamma_{j}, \lambda_{j k}\right)=f\left(\gamma_{j}, \lambda_{j k}\right)=\left\{a_{j k}\right\}
$$

and the proof is complete.

We note that, unlike the case of lattices mentioned above, the condition (9) is not necessary in general, even for sequences that lie on parallel lines. To see this, consider the weight $\varphi(z, w)=|z|^{2}+|z+w|^{2}$. Let $\Sigma=\{0\} \times \Gamma$, where $\Gamma$ is a sequence with density between $\frac{1}{2}$ and 1 . Then $\Gamma$ is interpolating in $W=\{0\} \times \mathbb{C}$ and $W$ is interpolating in $\mathbb{C}^{2}$. (In fact, the density of $W$ is zero.) But the reader can check that condition 
(9) does not hold. This observation suggests that perhaps the previously mentioned inductive approach is lacking another, possibly deep ingredient.

6.2. Application to sampling sequences. Let $\Sigma$ be a sequence of the form described before the statement of Theorem 3. By analogy with Theorem 3, we have the following application of Theorem 2 to sequences.

Theorem 4. Suppose that for some fixed $\varepsilon>0$, each $\Lambda_{j}$ has density $\geq 1+\varepsilon$ with respect to the weight $\varphi\left(\gamma_{j}, \cdot\right)$ and that, for some $r>0$,

$$
\frac{\# \Gamma \cap \mathbb{D}(z, r)}{r^{2} \Delta_{z} \varphi(z, w)}>\frac{\operatorname{det}(\sqrt{-1} \partial \bar{\partial} \varphi(z, w))}{\Delta_{z} \varphi(z, w) \Delta_{w} \varphi(z, w)}
$$

for all $z, w \in \mathbb{C}$. Then $\Sigma$ is sampling for $\mathfrak{B F}_{\varphi}^{p}\left(\mathbb{C}^{2}\right)$.

Proof. Let $W=\Gamma \times \mathbb{C}$. The upper sampling inequality holds since $W$ is uniformly flat and $\Sigma \subset W$ is uniformly separated on each line of $W$.

Next, let $F \in \mathfrak{B} \mathfrak{F}_{\varphi}^{p}\left(\mathbb{C}^{2}\right)$. Condition (10) implies that $W$ is sampling, and thus

$$
\int_{\mathbb{C}^{2}}|F|^{p} e^{-p \varphi} \omega^{2} \leq C_{1} \int_{W}|F|^{p} e^{-p \varphi} \omega .
$$

Now, since each $\Lambda_{j}$ is sampling with density bounded away from 1 uniformly in $j$, we see that there is $C>0$ such that for each $j$,

$$
\int_{\left\{\gamma_{j}\right\} \times \mathbb{C}}\left|F\left(\gamma_{j}, w\right)\right|^{p} e^{-p \varphi\left(\gamma_{j}, w\right)} d A(w) \leq C \sum_{k}\left|F\left(\gamma_{j}, \lambda_{j k}\right)\right|^{p} e^{-p \varphi\left(\gamma_{j}, \lambda_{j k}\right)} .
$$

Summing over $j$, we have

$$
\int_{W}|F|^{p} e^{-p \varphi} \omega \leq C_{2} \sum_{j, k}\left|F\left(\gamma_{j}, \lambda_{j k}\right)\right|^{p} e^{-p \varphi\left(\gamma_{j}, \lambda_{j k}\right)}
$$

This completes the proof.

\section{REFERENCES}

[BT-82] Berenstein, C.A.; Taylor, B.A., On the geometry of interpolating varieties. Sem. Lelong-Skoda 1980-81. Lecture Notes in Mathematics 919. Berlin, Heidelberg, New York: Springer 1982.

[Ber-83] Berndtsson, B., A Formula for Interpolation and Division. Math. Ann. 263 (1983), 399-418.

[BO-95] Berndtsson, B.; Ortega Cerdà, J., On interpolation and sampling in Hilbert spaces of analytic functions. J. Reine Angew. Math. 464 (1995), 109-128.

[Ber-97] Berndtsson, B., Uniform estimates with weights for the $\bar{\partial}$-equation. J. Geom. Anal. 7 (1997), no. 2, $195-215$.

[Ber-01] Berndtsson, B., Weighted estimates for the $\bar{\partial}$-equation. Complex analysis and geometry (Columbus, OH,1999), 43-57, Ohio State Univ. Math. Res. Inst. Plub., 9, de Gruyter, Berlin, 2001.

[Be-89] A. Beurling The collected works of Arne Beurling vol 2 Ed. L. Carleson et al., Birkhäuser, Boston pp. 341-365, 1989.

[B-64] Bishop, E., Conditions for the analyticity of certian sets. Michigan Math. J. 11 (1964), $289-304$.

[Ch-91] Christ, M., On the $\bar{\partial}$ equation in weighted $L^{2}$ norms in $\mathbb{C}^{1}$ J. Geom. Anal. 1 (1991), 193-230.

[Dem-82] Demailly, J.P., Scindage holomorhpe d'un morphisme de fibrés vectoriels semi-positifs avec estimation $L^{2}$. Sem. Lelong-Skoda 1980-81. Lecture Notes in Mathematics 919. Berlin, Heidelberg, New York: Springer 1982.

[FV-05] Forgacs, T., Varolin, D., Interpolating and Sampling for Weighted Bergman Spaces in the Unit Ball. Preprint 2005.

[Hor-90] Hörmander, L., An introduction to complex analysis in several variables. North-Holland, 1990.

[Lin-01] Lindholm, N., Sampling in weighted $L^{p}$ spaces of entire functions in $\mathbb{C}^{n}$ and estimates of the Bergman kernel. J. Funct. Anal. 182 (2001), no. 2, 390-426.

[MMO-03] Marco, N., Massaneda, X., Ortega-Cerdá J., Interpolating and sampling sequences for entire functions Geom. Funct. Anal., 13 (2003), 862-914.

[OS-98] Ortega-Cerdà, J.; Seip, K., Beurling-type density theorems for weighted $L^{p}$ spaces of entire functions. J. Anal. Math. 75 (1998), 247-266.

[SV-03] Schuster, A., Varolin, D., Generalized Bergman Spaces and Potential Theoretic Geometry of Open Riemann Surfaces. Preprint 2003. 
[Seip-92] Seip, K., Density theorems for sampling and interpolation in the Bargmann-Fock space. I. J. Reine Angew. Math. 429 (1992), 91-106.

[Seip-93] Seip, K., Beurling type density theorems in the unit disk. Invent. Math. 113 (1993), no. 1, 21-39.

[SW-92] Seip, K.; Wallstén, R. Density theorems for sampling and interpolation in the Bargmann-Fock space. II. J. Reine Angew. Math. 429 (1992), 107-113.

Departament de Matemàtica Applicada i AnÀlisi

Universitat de BARCElona, GRAN Via 585, 08071-BARCElona, SPAin

Email address: jortega@ub. edu

DEPARTMENT OF MATHEMATICS

SAN Francisco State University, SAN Francisco, CA 94132

Email address: schusteresf su.edu

DEPARTMENT OF MATHEMATICS

UNIVERSITY OF ILLINOIS AT URBANA-CHAMPAIGN, URBANA, IL 61801

Email address: dror@math.uiuc.edu 